\title{
Add-on effect of postural instructions to abdominopelvic exercise on urinary symptoms in climacteric women with stress urinary incontinence: A pilot randomized controlled trial.
}

\author{
Laura Fuentes Aparicio \\ Universitat de Valencia \\ Merce Balasch i Bernat ( $\nabla$ merce.balasch@uv.es) \\ Universitat de Valencia \\ Laura López Bueno \\ Universidad de Valencia
}

Research article

Keywords: Abdominal muscles, pelvic floor muscles, postural control, stress urinary incontinence, urinary symptoms, quality of life

Posted Date: April 30th, 2020

DOI: https://doi.org/10.21203/rs.3.rs-23923/v1

License: (c) (1) This work is licensed under a Creative Commons Attribution 4.0 International License.

Read Full License 


\section{Abstract}

\section{Background}

The aim of this study was to investigate the add-on effect of postural instructions to an abdominopelvic exercise program on urinary symptoms and QoL in perimenopausal, menopausal and postmenopausal women with stress urinary incontinence (SUI).

\section{Methods}

A randomized controlled trial was performed with a total of 40 perimenopausal, menopausal and postmenopausal women with SUI aged between 46 and 75 years old. Participants were randomly assigned to two groups: a group performing an abdominopelvic exercise program (AEP) $(n=20)$ and a group performing abdominopelvic exercise with the addition of postural instructions (AEPPI) $(n=20)$. Primary outcome measures were UI symptoms, Ul impact and QoL, measured by $48 \mathrm{~h}$ Pad Test and ICIQ$\mathrm{UI}$-SF, which were assessed at baseline, post-intervention and 3 months follow-up. Secondary outcome was patient's satisfaction measured by VAS scale only after the intervention.

\section{Results}

Between-groups differences were observed in terms of ICIQ-UI-SF scores inmediately after intervention. Within-groups differences were observed between baseline to 3 months follow-up and between postintervention to 3 months follow-up in AEPPI group $(p<0.05)$ for ICIQ-UI-SF and UI impact. UI symptoms were improved in both groups between baseline to 3-months follow-up $(p<0.05)$. Patient's satisfaction was higher in AEPPI group $(p<0.05)$.

\section{Conclusion}

The addition of postural instructions to an abdominopelvic exercise program improves QoL and patients' satisfaction in women with SUI.

Trial registration

This study was retrospectively registered at ClinicalTrials.gov under the number NCT03727945, https://clinicaltrials.gov/ct2/show/NCT03727945

\section{Background}

Urinary incontinence (UI) has been defined as the complaint of any involuntary leakage of urine ${ }^{1}$. UI has an average overall prevalence of $20-30 \%$. Its incidence increases with age, with an elevated prevalence in those aged 65 and over, causing loss of autonomy and quality of life $(\mathrm{QoL})^{2}$, feelings of distress, loss of self-esteem and social isolation ${ }^{3}$. Moreover, Ul leads to an important economic burden ${ }^{4}$. It is estimated that more than 2 million women are affected by some type of $\mathrm{UI}$, with stress urinary incontinence (SUI) 
being the most common among affected individuals ${ }^{56}$. SUl is characterized by involuntary loss of urine without any previous feeling of a need to void, which takes place on the occasion of a physical stress (cough, lifting something heavy, or any other physical activity). SUI can be manifested individually or in combination of urge urinary incontinence (UUI) in variable proportions (mixed urinary incontinence $(\mathrm{MUI}))^{1}$.

Pelvic floor muscles training (PFMT) has been recommended for all types of $\mathrm{Ul}^{7}$. Pelvic floor does not function as an independent entity; its function is also supported by other synergistic muscles ${ }^{8}$. In this regard, the relationship between PFM, deep erectors and deep abdominal muscles has been confirmed by electromiography ${ }^{9}{ }^{10}$. All these muscles are known as local system cavity ${ }^{8}$. A correct neuromuscular coordination of the trunk muscles would contribute to the maintenance of continence, by controlling intraabdominal and urethral closure pressure.

Current literature supports the use of a global approach in motor control exercise programs, including diaphragm, transversus abdominis (TrA) and PFM training ${ }^{8}$. The study of Fozzatti et al. $(2010)^{11}$ supports the use of this approach. They demonstrated that global postural reeducation can improve continence by normalizing diaphragmatic function and trunk stability. These muscles may play a key role in the prevention of SUI since a correct diaphragmatic breathing increases the antero-posterior diameter of the abdomen, which is believed to contribute to maintain the strength and resistance of the abdominal contraction during a sneeze $\mathrm{e}^{12}$.

Many studies have demonstrated the power and specificity of verbal instructions ${ }^{13} 14151617$. The single use of verbal instructions has been shown to have an impact on the distribution of activity in complementary muscles and postural muscles during different activities without any changes in the exercise performance ${ }^{14}$. More specifically, several authors have studied the effect of verbal instructions on pelvic floor exercises performance, these studies were based in the influence of proprioceptive input in muscle activity timing, coordination, balance and posture ${ }^{1516} 17$. In the study of Stafford et al. $(2016)^{16}$, the activation pattern of the PFM in a sample of men was influenced by verbal instructions. Likewise Vermandel et al. (2015) concluded that instructional feedback can improve PFM activation in women who initially were not able to perform a correct PFM contraction in early postdelivery.

However, it is still unknown how verbal instructions regarding posture may influence PFM activation and urinary continence during specific training of abdominopelvic muscles. Therefore, the aim of this study was to investigate the effectiveness of postural instructions added to abdominopelvic exercise program on the improvement of urinary symptoms and QoL in women with SUI.

\section{Method}

This pilot randomized controlled trial was carried out in the Functional Urology Unit at Dr. Peset University Hospital (Valencia) from 2014 to 2016. The study was approved by the Universitat de València Ethics Committee for Human Research (H1410616852782) and was retrospectively registered at 
ClinicalTrials.gov under the number NCT03727945. The research was conducted in accordance with the ethical principles established by the Declaration of Helsinki and CONSORT guidelines.

Women referred from urology consultations at the hospital were invited to participate in this research. Perimenopausal, menopausal and postmenopausal women aged between 40-75 years old who had SUI or stress-predominant mixed UI (MUI) were considered for inclusion. Perimenopausal period included the time immediately prior to the menopause and the first year after menopause ${ }^{18}$. The type of UI was diagnosed by experienced urologists, through both urodynamic and clinical assessment. Participants were excluded if they had grade 3-4 prolapse, functional impairment (Barthel scale $<85$ points), neurological or cognitive impairment (mini mental examination $<24$ points), or the presence of any other type of UI. Participation was voluntary, and all participants signed an informed consent prior to commencement of the study. Participants meting the eligibility criteria were allocated in two groups.

Intervention

Both groups performed 12 sessions lasting 40 minutes with a frequency of once a week ${ }^{19}$. The exercise programs were led by a physical therapist with 10 years of clinical experience in women's health. In the first session, postural pattern and pelvic floor muscle function were assessed. Then, women were taught how to contract PFM correctly. In the following sessions (2nd to 6th), a progressive specific pelvic floor muscle training was performed. In the 6th session, the participants were instructed on TrA activation, which was then added to the PFMT from 6th to 12th sessions. The degree of difficulty progressed according to different variables, such as the body position (supine decubitus, lateral decubitus, sitting ball, standing and functional tasks), the number of repetitions or the duration of contractions (Table 1).

All patients performed daily home training exercises during the treatment period and received a document including different abdominopelvic exercises.

In addition, prior to any specific training, participants from AEPPI group were instructed to maintain cervical alignment, scapular relocation and neutral pelvic tilt. These postural adjustments are supported by the biomechanical principles based in a "neutral spine", where function is maximized and the risk of injury is minimized ${ }^{20} 21$. The physical therapist provided both verbal and manual feedback in order to teach them the correct posture. In order to guide the postural correction, the physiotherapist gently pushed patients' chin while asking them to maintain cervical alignment. Then, she gently pushed their shoulders backwards to achieve scapular alignment, and their iliacs until the neutral pelvic tilt was reached. Then, participants were asked to practice 3 repetitions of this technique. During the AEP program, the physical therapist continued providing verbal and manual feedback if she deemed it necessary based on visual observation.

\section{Outcomes}

Data from all participants were collected at baseline, immediately after (post-intervention), and 3 months after the intervention (3 months follow-up). Evaluation was performed by the same physiotherapist. All 
patients completed a standard medical history questionnaire, including sociodemographic and clinical data. The type of UI was assessed according to clinical symptoms and urodynamic assessment.

Primary outcomes were UI symptoms, UI impact and ICIQ-UI-SF scores. Ul symptoms were quantified through the amount of urine loss, which was measured using a $48 \mathrm{~h} \mathrm{Pad} \mathrm{Test}^{1}$. This test is a standardized method for quantifying urine leakage that can be performed at home ${ }^{22}$. Patients use and replace pads according to their needs over $48 \mathrm{~h}$. Results are calculated by the difference between the net weight of the used pads and the sum of the non-used ones. The score of the Pad Test can be interpreted as mild (4-20 g), moderate $(21-74 \mathrm{~g})$ and severe $\mathrm{UI}(>75 \mathrm{~g})^{23} 24$.

Ul impact and QoL were measured using the spanish validation of the International Consultation Incontinence Short-Form (ICIQ-UI-SF) ${ }^{25}$. This questionnaire is highly recommended for the evaluation of urinary incontinence symptoms in women (grade A) ${ }^{26} 27$. Furthermore, the assessment of QoL has been recommended by the International Continence Society (ICS) as a complement to clinical measures for the evaluation of $\mathrm{UI}^{28}$.

The ICIQ-UI-SF is made up of 3 questions assessing frequency of the leaks, amount of leakage, and impact on QoL. This questionnaire also comprises a fourth non-scored item to assess patients' perception regarding the cause and type of leakage. This item is specially useful in a clinical context. The final score results in a sum of the 3 questions, ranging from 0 to 21, with a higher score indicating more severe Ul and greater impact on QoL 252627.

Secondary outcome was patient's satisfaction measured using a 100-point Visual Analogue Scale $(\text { VAS })^{29}$ after intervention. In our study, higher scores indicate more satisfaction with treatment.

In order to calculate the sample size, an a priori power analysis was conducted using G*Power software (version 3.1.9.2). Assuming an analysis of variance (ANOVA) of repeated measurements, a medium effect size $\left(d=0.5 ; \eta_{p}^{2}=0.06\right), a=0.05$, power $=0.90$, and a correlation among repeated measurements of 0.5 , a total sample size of 36 participants would be needed to achieve an appropriate power level for this research.

Participants were randomly allocated to two different groups: the first one performed an abdominopelvic exercise program (AEP) $(n=20)$, and a second one in which the subjects underwent the same exercise program with the addition of postural instructions (AEPPI) $(n=20)$. The allocation of the subjects to either the AEP or AEPPI group was based on the output of a random number generator program the software Research Randomizer (Version 4.0). The research team was composed by 3 urologists and a physiotherapist with 20 years of experience. It was not possible to blind the care providers, since it was the physiotherapist who carried out both interventions. Likewise, participants were not blinded as they had been provided with an information brochure explaining the possible interventions. An external assessor was in charge of randomization and was blinded to the concealment of group allocation, as these were numerically coded. 
For the statistical analysis, the statistics package SPSS 24.0.0 was used. Data normality was explored using the Shapiro-Wilk test. Descriptive statistics were used to present sociodemographic and clinical data. Subjects' characteristics were compared using Student's $t$ - or Chi-Square tests. Mixed 2-factor ANOVAs with repeated-measures in the time factor were used to determine significant differences between groups (AEP and AEPPI) and time point (baseline, post-intervention and 3 months follow-up) for the UI symptoms, UI impact and ICIQ-UI-SF total scores outcomes. Post hoc analysis with the Bonferroni correction was used for the multiple comparison tests. Moreover, a Student's $t$-test was used for comparing patient's satisfaction. Effect size was interpreted as small $(d=0.2)$, medium $(d=0.5)$ and large $(d>0.8)$. The significance level was set at 0.05 .

\section{Results}

A total of 47 women with SUI were included in the trial and received either an abdominopelvic exercise program (AEP, $n=23$ ), or an AEP combined with postural instructions (AEPPI, $n=24)$. Flow diagram depicts the recruitment and retention of participants in this trial (Fig. 1). Three participants in AEP group and 4 in AEPPI group dropped out the study. The total sample consisted of 40 women aged between 40 and 75 years old, with a mean age of 59.47 (9.34) years. There were no statistically significant differences regarding sociodemographic and clinical variables between two groups (Table 2). All participants complied $100 \%$ of the exercise program.

Primary outcomes

ANOVA analysis showed that there were no statistically significant differences between the AEP and AEPPI groups for any variable at baseline $(p>0.05)$ (Table 3$)$.

Between-group analysis showed significant differences for the ICIQ-UI-SF score measured after the intervention, showing higher improvement for the AEPPI compared to the AEP group (1.29 points).

Within-group analysis showed non-significant differences between baseline and post-intervention for either AEP or AEPPI group. However, when comparing post-intervention and 3 months follow-up, AEPPI group obtained a significant reduction in ICIQ-UI-SF (1.75 points), whereas UI symptoms (48 h Pad Test) and UI impact remained unchanged for both groups. Regarding the change between baseline and 3 months follow-up, significant reductions were observed in UI symptoms (48 h Pad Test) for both groups (23.92 $\mathrm{g}$ in AEP group and $24.45 \mathrm{~g}$ in AEPPI group), while a decrease was observed in ICIQ-UI-SF scores (3.80 points) and UI impact (0.75 points) for AEPPI group.

\section{Secondary outcomes}

We found significant differences in patients' satisfaction between groups $(p=.021)$, in favor of the AEPPI group, who reported higher values (91.5 points) compared to the AEP group (85.5 points). 


\section{Discussion}

Main findings

Several studies have previously investigated the role of posture in continence. However, none of them have measured the effect of postural instructions on urinary continence. The present study demonstrated that a 12-sessions abdominopelvic exercise program supplemented with postural instructions can improve QoL and satisfaction in women with SUI.

Despite slight differences between both groups were observed for Ul symptoms (48 h Pad test) and UI impact, these were not statistically significant. The findings of the present study suggest that both $\mathrm{UI}$ symptoms and $\mathrm{UI}$ impact show a trend to be reduced when postural instructions are added to an abdominopelvic training program. It is important to note that both AEP and AEPPI groups showed an improved rate for Ul symptoms (reduction in UI symptoms severity) and UI impact after completion of the intervention. We found a progressive orine leakages reduction in both groups AEP and AEPPI groups $(53.80 \mathrm{~g} \pm 78.57 \mathrm{~g}$ to $29.88 \mathrm{~g} \pm 51.01 \mathrm{~g}$ and $40.70 \mathrm{~g} \pm 44.30 \mathrm{~g}$ to $16.25 \mathrm{~g} \pm 24.53 \mathrm{~g}$ ), respectively, from baseline to 3 months follow-up. Regarding UI impact, although not significant, AEPPI group showed a greater reduction (7.5\%) compared to the AEP group (3.7\%) from baseline to 3 months follow-up. Similar results were obtained by Hirakawa et al. ${ }^{30}$, who compared the effects of PFM training with and without biofeedback in a sample of women with SUI. In this study, the leakage volume measured by the $1 \mathrm{~h}$ Pad Test tended to decrease in both groups after 12 weeks of training, but this effect was not significant. Other authors ${ }^{31}$ have found a significant improvement in the amount of leakage measured by de 20 min Pad Test when comparing an intervention group with a control group after 12 weeks based on diaphragmatic and abdominopelvic training. In our study, although baseline severity of the Ul symptoms did not significantly differ between groups, the two samples were not strictly homogeneous, since participants in the AEP group presented more severe UI symptoms than women in the AEPPI group. We believe that this fact could have influenced the different recovery rate of participants. It may be that the minor improvement experienced by the participants from the AEPPI group has been influenced by the fact that they initially presented less severe UI symptoms, leading to lower chances of improving. Therefore, a longer intervention (more than 3 months) may be necessary for women with less severe symptoms to achieve an important improvement. On the other hand, could be that women with more severe UI symptoms can benefit more from this program. Regarding QoL and patients' satisfaction, AEPPI reported/showed greater improvement (reduction of 18\% and $91.5 \%$ in ICIQ-UI-SF and patient's satisfaction scores, respectively) compared to AEP group (reduction of $7 \%$ and $85.5 \%$ in ICIQ-UI-SF and patient's satisfaction scores, respectively) immediately after treatment, suggesting that women with SUI performing abdominopelvic exercise supplemented with postural instructions experienced better QoL and satisfaction compared to those performing the abdominopelvic exercise program without postural instructions. Despite these findings, this study failed to find between-group differences in the mid-term for QoL (3 months follow-up). This result could be considered reasonable, since after the 12 weeks training program no other intervention or home-based exercise was recommended to the participants. 
It is necessary to highlight the importance of the findings of the present study since the effect of UI on QoL is as or more important as Ul symptoms, according to previous literature. ${ }^{22} 242829$ No correlation has been observed between objective and subjective assessments in patients with Ul. Thus, individual perception is not directly linked to objective measures that quantify the amount of urinary loss.

Further, the number of previous studies that analyzed the impact of postural instructions in abdominopelvic training in patients with $\mathrm{Ul}$ is limited. Currently, there is a wide variety of abdominopelvic exercise protocols used for the treatment of UI. Different studies have investigated the effect of these interventions on $\mathrm{Ul}$ impact and QoL, and verbal or tactile feedback concerning to correct posture are frequently provided in their training protocols. To our knowledge, no previous studies evaluated the addon effect of postural instructions to abdominopelvic training.

Several studies have evaluated the effect of abdominopelvic training in women with UI. Some authors have compared two active groups ${ }^{11} 323334$ while others have compared abdominopelvic groups versus a control $^{31} 35$. The studies comparing two active intervention groups reinforce the idea that global abdominopelvic training improves UI symptoms ${ }^{32} 33$ and general health perception ${ }^{34}$. Fozzatti et al. $(2010)^{11}$ evaluated the impact of Global Postural Re-education on SUI symptoms and compared it to PFM training, using another QoL questionnaire, King's Health Questionnaire (KHQ). In this study, no differences were detected between groups, suggesting that postural global training, even though not including specific exercises for the PFM, brings comparable benefits to isolated PFM training in patients' QoL. Gadheri et al. (2016) ${ }^{33}$ compared the effect of a group of patients performing stabilization exercise with a group performing the same exercise focusing on PFM. The results of this study differ from our results, showing no differences between both groups regarding QoL, measured by ICIQ-UI-SF. These findings highlight the relevance of different training methods not only focused on PFM. Moreover, Ozengin et al. (2015) ${ }^{34}$ detected an increase in general health perception, measured by Prolapse Qualityof-life Questionnaire (P-QOL), in a group performing stabilization exercises compared to PFM training, in women with stage 1 and 2 pelvic organ prolapse. More recently, Ptak et al. (2017) ${ }^{32}$ revealed that PFM with additional exercise for the TrA muscle was shown to be more effective than isolated PFM exercise in most QoL domains, measured with the International Consultation on Incontinence Modular Questionnaire-Lower Urinary Tract Symptoms quality of life (ICIQ LUTS) QoL, based in the KHQ. On the other hand, the studies comparing abdominopelvic exercise with a control group suggest that this training method improves the urinary symptoms $s^{113136}, \mathrm{QoL}^{35}$ and muscle function ${ }^{36}$. Hung et al. $(2010)^{31}$ reported improvement of both QoL and UI impact in the training group, based on diaphragmatic, deep abdominal and PFM coordinated function, compared to a control group. In contrast to our study, these outcomes were measured by the Symptom Impact Index questionnaire. Also Alves et al. $(2015)^{35}$ found decreased urinary symptoms based on ICIQ-UI-SF in the group of participants performing abdominopelvic exercise. Finally, Tajiri et al. $(2014)^{36}$ also reported improved Ul symptoms following abdominopelvic training as well as an enhanced muscle function, with an increased TrA muscle thickness. Taken together, these results reinforce the idea that global approaches are an appropriate intervention for the treatment of patients presenting UI. 
Regarding patient's satisfaction with the training protocol, the results of our study showed that patients from the AEPPI group were more satisfied, based on VAS, than participants from the AEP group. Previous research ${ }^{35}$ has already demonstrated higher values of satisfaction with treatment when comparing abdominopelvic exercise with a control group.

Some studies have demonstrated that patients' satisfaction is related to adherence to treatment ${ }^{37}$. In particular, it has been stated that perception of the effectiveness of the treatment is considered as a determinant factor contributing to adherence. This issue becomes especially relevant in those treatment programs that require an active role and involvement of the patient to obtain benefits from the therapy.

Strengths and limitations

To our knowledge, this study is the first pilot randomized, controlled trial to evaluate the effectiveness of abdominopelvic training supplemented with postural instructions in women with SUI. In addition, in this study the abdominopelvic exercise program, which was supervised by a physical therapist, was reinforced by the performance of home training exercises. The results presented are promising since participants receiving postural instructions achieved better outcomes in QoL and patients' satisfaction. Moreover, although not significantly, Ul symptoms and UI impact were also improved when postural instructions were added to the abdominopelvic program. According to the findings of this study, the implementation of postural instructions regarding spine, scapula and pelvis posture should be highly encouraged amongst physical therapists in the context of abdominopelvic exercise programs performance in their daily clinical practice.

However, this study has several limitations. First, could be a possible limitation in relation to the generalizability of the current study was the small sample size, although the number of recruited participants was sufficient in accordance with an a priori power analysis.

Additionally, further research with higher sample sizes would help in generalizing the findings of this study. Second, the assessment method for Ul symptoms in regard to measuring the urinary loss based on pads' weight (according to the $48 \mathrm{~h}$ Pad Test) might also be a study limitation. Third, participants' adherence to home training exercises was not registered or considered in this study, and this could have biased our results. Despite the findings of this research show the effectiveness of adding of postural instructions to abdominopelvic exercises in terms of QoL and patients' satisfaction over time, it would be interesting to investigate the effects of such intervention in the longer term after the completion of the intervention (i.e. 6 months and 12 months-follow up).

According to the results of this study, it is plausible that the addition of exercises more specifically addressed to the PFM activation to the proposed abdominopelvic exercise program could have improved the outcomes related to Ul symptoms and UI impact in a sample of women with SUI, when supplementing their exercise program with postural instructions. Besides, further research on how the activation of PFM and abdominopelvic muscles is affected by different instructions is required in order to know whether instructions enhance the influence the activation pattern (EMG activity) of different PFM. This would also 
allow to determine whether better outcomes can be achieved with instructions tailored to the women continence mechanism. It would be also interesting to find out which postural instructions are more effective for enhancing PFM contraction and UI symptoms in women with SUI. Future studies focusing in the comparison/ comparing the effect of different postural instructions on QoL and Ul symptoms may help to determine the most appropriate postural approach in this population. Moreover, it could be interesting to investigate the effect of a more extended program, including home-based exercises focused in postural alignment after the 12-week abdominopelvic program proposed in the present study, which may lead to obtain better mid-term outcomes.

\section{Conclusions}

Both an isolated program of abdominopelvic exercises and a combined program of abdominopelvic exercise supplemented by postural instructions were effective for the improvement of Ul symptoms, QoL and patients' satisfaction in a sample of climacteric women with SUI. The abdominopelvic training program with postural instructions was more effective than abdominopelvic training alone in enhancing QoL and patients' satisfaction immediately after intervention. Moreover, the combined program may have a greater potential for the improvement of UI symptoms and UI impact, but this effect was not demonstrated in this study.

\section{Abbreviations}

QoL

quality of life

UI

urinary incontinence

SUI

stress urinary incontinence

AEP

abdominopelvic program exercise

AEPPI

abdominopelvic exercise with the addition of postural instructions

ICIQ-UI-SF

International Consultation Incontinence Short-Form

ICS

International Continence Society

VAS

Visual Analogue Scale

UUI

urge urinary incontinence

MUI 
mixed urinary incontinence

PFMT

pelvic floor muscles training

PFM

pelvic floor muscles

TrA

tranversus abdominis

$\mathrm{KHQ}$

King's Health Questionnaire

P-QoL

Prolapse Quality-of-life Questionnaire

ICIQ-Luts

Modular Questionnaire-Lower Urinary Tract Symptoms quality of life

EMG

electromyography

\section{Declarations}

Ethics approval and consent to participate

The present study was approved by the Universitat de València Ethics Committee for Human Research (H1410616852782). Verbal and written information about the study were provided to the participants. Written consent for participation was obtained from the participants prior to the beginning of the intervention.

Consent of publication:

Patient's information was coded in order to avoid the possiblity of recognising any individual person during interpretation of data or presentation of results in the manuscript. The participants received information about this procedure before they provided written consent to participate and consent for publication, which included image consent.

Availability data and materials

The datasets used and/or analysed during the current study are available from the corresponding author on reasonable request.

Competing interests

The authors declare that they have no competing interests.

Funding 
None

Authors' Contributions

All authors have substantially contributed to all parts of the study: the study design, acquisition and analysis of data, interpretation of results and drafting of the manuscript. LF and LL shared responsibility for data collection, performing data analyses and interpretation of data and results. LF had the principal responsibility for drafting the manuscript, and MB and LL contributed to the drafting of the text. All authors have read and approved the final manuscript and are accountable for all aspects of the work.

Acknowledgements

We acknowledge the urologists from the Functional Urology Unit of Dr. Peset University Hospital (Valencia), who contributed to patients' recruiment.

\section{References}

1. Haylen BT, de Ridder D, Freeman RD, Swift SE, Berghmans B, Lee J, et al. An International Urogynecological Association (IUGA)/International Continence Society (ICS) joint report on the terminology for female pelvic floor dysfunction. Int Uroginecol. 2010;21(1):5-26; doi:10.1007/s00192009-0976-9.

2. Salinas Casado J, Díaz Rodríguez A, Brenes Bermúdez F, Cancelo Hidalgo MJ, Cuenllas Díaz A, Verdejo Bravo C. Prevalencia de la incontinencia urinaria en España. Urod A. 2010;23(1):52-66.

3. Aguilar-Navarro SG, Incontinencia urinaria en el adulto mayor. Rev Enferm Inst Mex Seguro Soc. 2007;15(1):51-56.

4. Zunzunegui Pastor MV, Rodríguez Laso A, García de Yébenes MJ, Aguilar Conesa M.D, Lázaro y de Mercado P y Otero Puime A. Prevalencia de la incontinencia urinaria y factores asociados en varones y mujeres de más de 65 años. Aten Primaria. 2003;32(6):337-342; doi:10.1016/s02126567(03)79293-2.

5. Grosse D, Sengler J. Reeducación del periné fisioterapia en las incontinencias urinarias. Barcelona. Ed Masson; 2001.

6. Robles JE (editor). I Curso de formación en incontinencia urinaria. Newbook ediciones; 2001.

7. Guía clínica sobre la incontinencia urinaria. Schroder A, Abrams P. Andersson KE, Artibani W, Chapple CR, Drake MJ, Hampel C, Neisius A, Tubaro A, Thuroff JW. European Association of Urology. 2010.

8. Sapsford R. The pelvic floor. A clinical model for function and rehabilitation. Physiotherapy. 2001;87(12):620-630. 
9. Richardson C, Jull G, Hodges P, Hides J. Therapeutic exercise for spinal segmental stabilization in low back pain. 2nd London: Churchill Livingstone, Edinburgh; 1999.

10. Neumann P, Gill V. Pelvic floor and abdominal muscle interaction: EMG activity and intra- abdominal pressure. Int Urogynecol J. 2002;13(2):125-132; doi 10.1007/s001920200027.

11. Fozzatti C, Herrmann V, Palma T, Riccetto CL, Palma PC. Global Postural Re-education: an alternative approach for stress urinary incontinence? Eur J Obstet Gynecol Reprod Biol. 2010;152(2):218-224; doi:10.1016/j.ejogrb.2010.06.002.

12. R. Rehabilitation of pelvic floor muscles utilizing trunk stabilization. Man Ther. 2004;9:3-12; doi:10.1016/s1356-689x(03)00131-0.

13. Karst, GM and Willett, GM. Effects of specific exercise instructions on abdominal muscle activity during trunk curl exercises. J Orthop Sports Phys Ther. 2004;34:4-12; doi:10.2519/jospt.2004.34.1.4.

14. Sahaly, R, Vandewalle, H, Driss, T, and Monod, H. Surface electromyograms of agonist and antagonist muscles during force development of maximal isometric exercises-effects of instruction. Eur J Appl Physiol. 2003;89:79-84; doi:10.1007/s00421-002-0762-6.

15. Kharaji G, Afsaneh Nikjooy A, Amiri A, Anjari MA. Proprioception in stress urinary incontinence: A narrative review. Med J Islam Repub Iran. 2019;33:1-5; doi: 10.34171/mjiri.33.60.

16. Stafford R, Constantinou C, Asthon-Miller J, Hodges P. Pattern of activation of pelvic floor muscles in men differs with verbal instructions: Pattern of Activation of Pelvic Floor Muscles. Neurol Urodyn. 2015;35(4):457-463; doi:10.1002/nau.22745.

17. Vermandel A, De Wachter S, Beyltjens T, D`Hondt D, Jacquemyn Y, Windaele JJ. Pelvic floor awareness and the positive effect of verbal instructions in 958 women early postdelivery. Int Urogynecol J. 2015;26(2):223-228; doi:10.1007/s00192-014-2483-x.

18. Utian WH. The International Menopause menopause-related terminology definitions. Climacteric.1999;2(4):284-286; doi:10.3109/13697139909038088.

19. Dumoulin C. Determining the optimal pelvic floor muscle training regimen for women with stress urinary incontinence. Neurourol Urodyn. 2011;30(5):746-753; doi:10.1002/nau.21104

20. Kiefer, A. Shirazi-Adl, M. Parnianpour. Stability of the human spine in neutral postures.Eur Spine J. 1996;6:45-53; doi:10.1007/bf01676574.

21. Oleksy $Ł$, Mika A, Kielnar R, Grzegorczyk J, Marchewka A, Stolarczyk A., et al. The influence of pelvis reposition exercises on pelvic floor muscles asymmetry. Medicine. 2019;98(2):1-7; doi:10.1097/MD.0000000000013988. 
22. Krhut J, Zachoval R, Smith PP, Rosier FWM P, Valasky L, Martan A, et al. Pad weight testing in the evaluation of urinary incontinence. Neurol Urodyn. 2014;33(5):507-10; doi: 10.1002/nau.22436.

23. Ferreira CHJ, Bo K. The Pad Test for urinary incontinence in women. J Physiother. 2015; 61(2):98; doi: 10.1016/j.jphys.2014.12.001.

24. Matharu GS, Assassa RP, Williams KS, Donaldson M, Matthews R, Tincello DG. Objective Assessment of Urinary Incontinence in Women: Comparison of the One-Hour and 24-Hour Pad Tests. Eur Urol. 2004;45(2):208-212; doi:10.1016/j.eururo.2003.09.006.

25. Espuña Pons M, Rebollo Álvarez P, Pablo Rebollo Álvarez, Puig Clota M. Validación de la versión española del International Consultation on Incontinence Questionnaire Short Form. Un cuestionario para evaluar la incontinencia urinaria. Med Clin. 2004;122(8):288-292; doi: 10.1016/s00257753(04)74212-8.

26. Avery K, Donovan J, Peters J, Shaw C, Gotoh M, Abrams P. ICIQ: A brief and robust measure for evaluating the symptoms and impact of urinary incontinence. Neurourol Urodyn. 2004; 23 (4):322330; doi:10.1002/nau.20041.

27. Abrams P, Avery K, Gardener N, et al. The International Consultation on Incontinence Modular Questionnaire. www.iciq.net. J Urol. 2006;175:1063-1066; doi:10.1016/S0022-5347(05)00348-4.

28. Gould D, Kelly D, Goldstone L, Gammon J. Visual Analogue Scale (VAS). J Clin Nurs. 2001; 10:697706.

29. Dauphin AP, Guillemin F, Virion JM, Briançon S. Bias and Precision in Visual Analogue Scales: A Randomized Controlled Trial. Am J Epidemiol. 1999;150(10):1117-1127; doi:

10.1093/oxfordjournals.aje.a009937.

30. Hirakawa T, Suzuki S, Kato K, Gotoh M, Yoshikawa Y. Randomized controlled trial of pelvic floor muscle training with or without biofeedback for urinary incontinence. Int Urogynecol. 2013;24(8):1347-1354; doi:10.1007/s00192-012-2012-8.

31. Hung HC, Hsiao S M, Chilh SY, Lin HH, Tsaou JY. An alternative intervention for urinary incontinence: retraining diaphragmatic, deep abdominal and pelvic floor muscle coordinated function. Man Ther. 2010;15(3):273-279; doi:10.1016/j.math.2010.01.008.

32. Ptak M, Brodowska A, Ciécwiez S, Rotter I. Quality of life in women with stage I stress urinary incontinence after application of conservative treatment. A randomized trial. J. Environ. Res. Public. Health. 2017;14(6):577-587; doi:10.3390/ijerph14060577.

33. Ghaderi F, Mohammadi K, Amin Sasar R, Niko Keslat S, Oskouei AE. Effects of Stabilization Exercises Focusing on Pelvic Floor Muscles on Low Back Pain and Urinary Incontinence in Women. Urol. 2016;93:50-54; doi:10.1016/j.urology.2016.03.034 
34. Ozengin N, Ün Yıldırım N, Duran B. A comparison between stabilization exercises and pelvic floor muscle training in women with pelvic organ prolapse. J Turk Soc Obstet Gynecol. 2015; 1:11-7; doi:10.4274/tjod.74317.

35. Alves FK, Riccetto C, Adami DBV, Marques J, Pereira LC, Palma P, Botelho S. A pelvic floor muscle training program in postmenopausal women: A randomized controlled trial. Maturitas. 2015;81(2):300-305; doi:10.1016/jmaturitas.2015.03.006.

36. Tajiri K, Huo M, Maruyama H. Effects of Co-contraction of Both Transverse Abdominal Muscle and Pelvic Floor Muscle Exercises for Stress Urinary Incontinence: A Randomized Controlled Trial. J Phys Ther Sci. 2014;26(8):1161-1163; doi:10.1589/jpts.26.1161.

37. Frawley HC, McClurg D, Mahfooza H, Hay-Smith J, Dumoulin C. Health professionals' and patients' perspectives on pelvic floor muscle training adherence-2011 ICS State-of-the-Science Seminar research paper IV of IV. Neurourol Urodyn. 2015;34(7):632-639; doi: 10.1002/nau.22774.

\section{Tables}

Table 1. Abdominopelvic exercise program

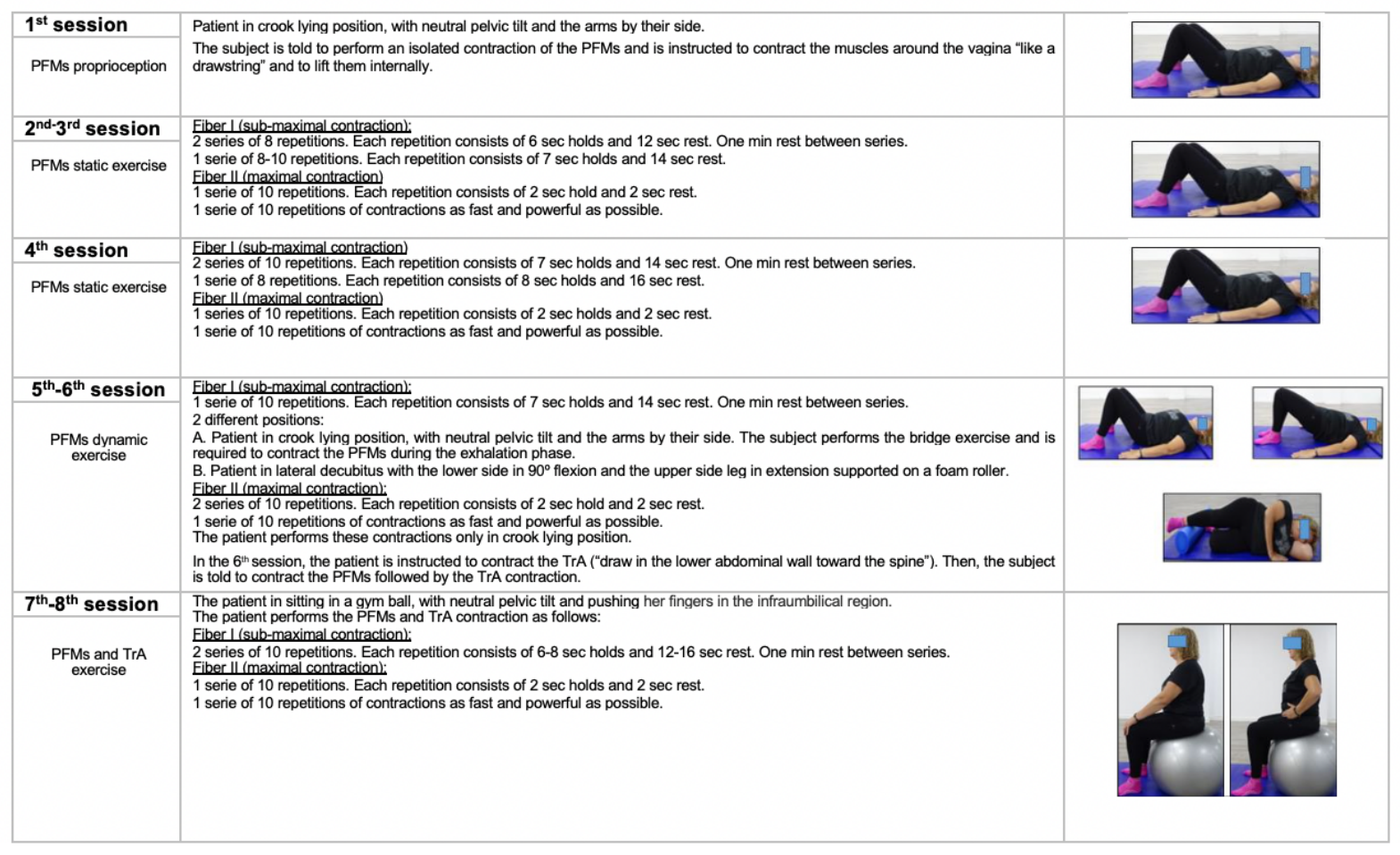




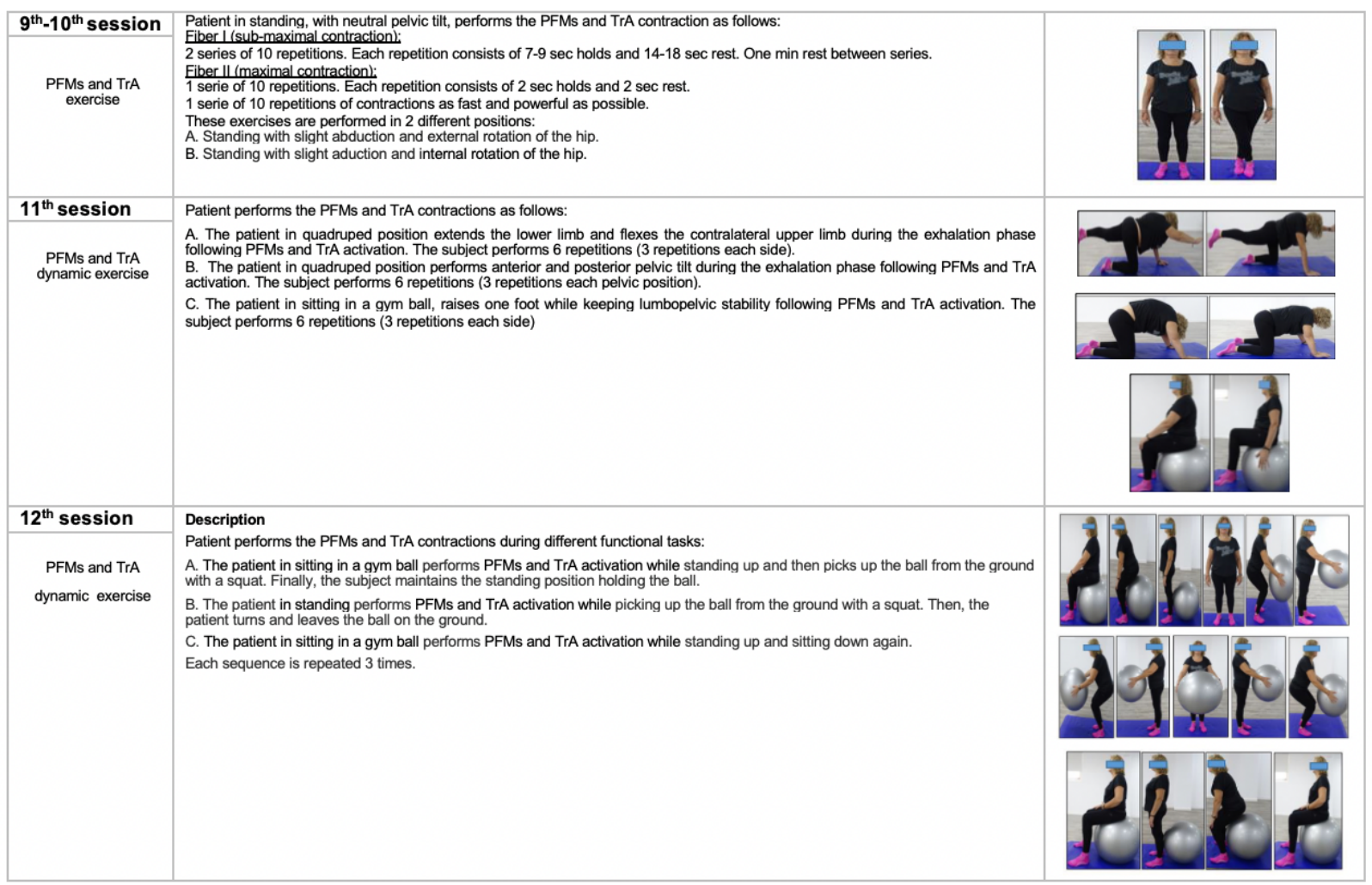

PFMs, pelvic floor muscles; TrA, tranversus abdominis.

Table 2. General sociodemographic and clinical data $(n=40)$ 


\begin{tabular}{|c|c|c|c|}
\hline & $\operatorname{AEP}(n=20)$ & AEPPI $(n=20)$ & $p$-Value \\
\hline Age (years) - mean $S D$ & $61.95(9.34)$ & $57(8.88)$ & $.094^{*}$ \\
\hline IBM kg/m² - mean $S D$ & 27.68(6.07) & $27.70(6.63)$ & $.984 *$ \\
\hline Menopause age - mean $S D$ & $48.47(4.87)$ & $48.47(4.87)$ & $.850 *$ \\
\hline Evolution time-mean $S D$ & $8.1(9.49)$ & 8.3(6.68) & $.939 *$ \\
\hline Hysterectom $y-n(\%)$ & $8(40 \%)$ & $4(20 \%)$ & $.176^{* *}$ \\
\hline Type UI & & & $478^{* *}$ \\
\hline $\mathrm{SUI}-n(\%)$ & $14(70 \%)$ & $16(80 \%)$ & \\
\hline MUI - $n(\%)$ & $6(30 \%)$ & $4(20 \%)$ & \\
\hline \multicolumn{4}{|l|}{ Obstetric history - mean $S D$} \\
\hline Parity & $1.47(.009)$ & $2.00(1.45)$ & $.193^{*}$ \\
\hline Cesarean & $0.10(0.30)$ & $0.20(.41)$ & $.389 *$ \\
\hline$\underline{\text { Hormone replacement }-n(\%)}$ & $7(35 \%)$ & $4(20 \%)$ & $.300^{* *}$ \\
\hline Hormonal status - $n$ (\%) & & & $.235^{* *}$ \\
\hline Perimenopause & $3(15 \%)$ & $5(20 \%)$ & \\
\hline Menopause & $5(25 \%)$ & $7(25 \%)$ & \\
\hline Postmenopause & $12(60 \%)$ & $8(35 \%)$ & \\
\hline Physical activity $-n$ (\%). & & & $.211^{* *}$ \\
\hline High impact & $3(15 \%)$ & $4(20 \%)$ & \\
\hline Low impact & $13(65 \%)$ & $9(45 \%)$ & \\
\hline No activity & $4(20 \%)$ & $7(35 \%)$ & \\
\hline
\end{tabular}

*Student's $t$-test

**Chi-Square-Statistic.

Data are expressed as mean (standard desviation).

AEP, Abdominopelvic exercise program; AEPPI, Abdominopelvic exercise program adding postural instructions; IBM, body max index; UI, urinary incontinence; SUI, stress urinary incontinence; MUI, mixed urinary incontinence.

Table 3. Results of the comparison for the different variables studied 


\begin{tabular}{|c|c|c|c|c|c|c|c|}
\hline & Group & Baseline & $\begin{array}{l}\text { Post- } \\
\text { intervention }\end{array}$ & $\begin{array}{c}3 \mathrm{~m} \\
\text { Follow-up }\end{array}$ & $\begin{array}{l}\text { Comparisons } \\
\text { Baseline- } \\
\text { post } \\
\\
\quad p^{a}[95 \%\end{array}$ & $\begin{array}{c}\text { Comparisons } \\
\text { Post- 3m follow- } \\
\text { up } \\
\text { p }^{\mathrm{a}}[95 \% \mathrm{CI}] \mathrm{hp} 2\end{array}$ & $\begin{array}{c}\text { Comparisons } \\
\text { Baseline- 3m } \\
\text { Follow-up } \\
\text { p }^{\mathrm{a}}[95 \% \mathrm{CI}] \mathrm{hp} 2\end{array}$ \\
\hline & & & & & CI] hp2 & & \\
\hline \multirow{4}{*}{$\begin{array}{l}\text { Urinary } \\
\text { simptoms }\end{array}$} & AEP & $53.80(78,57)$ & $36.13(59.05)$ & 29.88 (51.01) & .084 [37.07 to & .700 [19.17 to & $.024^{b}[45.31$ to \\
\hline & AEPPI & $40.70(44.30)$ & $27.43(35.75)$ & $16.25(24.53)$ & $-1.73] ; 0.467$ & $-6.68] ; 0.537$ & $2.52] ; 0.632$ \\
\hline & $\mathrm{P}^{\mathrm{C}}[95 \%$ & $.522[54.21$ to & .579 [40.18 to & .291 [39.39 to & $.260[32.17$ to & .097 [23.78 to & $.017^{b}[45.32$ to \\
\hline & $\mathrm{CI}] ; \mathrm{hp} 2$ & -28.00]; .011 & -22.79]; .008 & $-12.12] ; .030$ & $-5.64] ; 0.351$ & $-1.41] ; 0.557$ & 3.59]; 0.727 \\
\hline \multirow[t]{4}{*}{ UI impact } & AEP & $2.10(.875)$ & 1.84(898) & $1.73(.805)$ & $.597[.242$ to & $1.00[.552$ & $0.83[.772$ to \\
\hline & AEPPI & $2.60(.882)$ & $2.20(.894)$ & $1.85(.745)$ & $-.768] ; 0.271$ & to.342]; 0 & $.035] ; 0.001$ \\
\hline & $\mathrm{P}^{\mathrm{C}}[95 \%$ & .087 [1.065 to & $.220[.224$ to & .651 [.390 to & $.146[.892$ to & .154 [.786 to & $.000^{\mathrm{b}}[1.143$ to \\
\hline & $\mathrm{CI}] ; \mathrm{hp} 2$ & $-0.76] ; .077$ & $-.940] ; .040$ & $-.6116] ; .006$ & $-.092] ; 0.531$ & $-.086] ; 0.531$ & $.357] ; 0.962$ \\
\hline \multirow{4}{*}{$\begin{array}{l}I C I Q-U I- \\
S F\end{array}$} & AEP & $11,10(4.21)$ & $10.36(3.71)$ & $9,63(3.54)$ & $1.00[3.15$ to & $1.00[2.69$ to & .056 [2.97 to \\
\hline & AEPPI & $13.70(4.00)$ & $11.65(4.88)$ & $9,90(3.44)$ & -1.67]; 0.160 & $-1.21] ; 0.193$ & $-0.28] ; 0.635$ \\
\hline & $\mathrm{P}^{\mathrm{C}}[95 \%$ & $.056[.071$ to & $.042^{\mathrm{C}}[1.44$ to & $.812[2.538$ to & $.210[4.10$ to & $.031^{b}$ [3.95 to & $.000^{b}[5.26$ to \\
\hline & $\mathrm{CI}] ; \mathrm{hp} 2$ & $-5.260] ; .095$ & $-4.14] ; .025$ & $-2,001] ; .002$ & $-.603] ; 0.610$ & $.146] ; 0.678$ & 2.33]; 1.359 \\
\hline
\end{tabular}

Data are expressed as mean (Standard desviation)

$\eta_{\mathrm{p}}{ }^{2}$, Partial eta squared; 95\% CI, 95\% confidence interval; AEP, Abdominopelvic exercise program; AEPPI, Abdominopelvic exercise program adding postural instructions; UI symtpoms based in 48h Pad Test registered.

${ }^{\mathrm{a}}$ Corresponds to the differences between baseline and post-intervention/ post-intervention and 3 months follow-up/ baseline and 3 months follow-uo

${ }^{\mathrm{b}}$ Indicates significant difference

${ }^{\mathrm{c}}$ Corresponds to the differences between AEP and AEPPI.

\section{Figures}




\section{O N S O R T}

TRANSPARENT REPORTING of TRIALS

\section{CONSORT 2010 Flow Diagram}

\section{Enrollment}

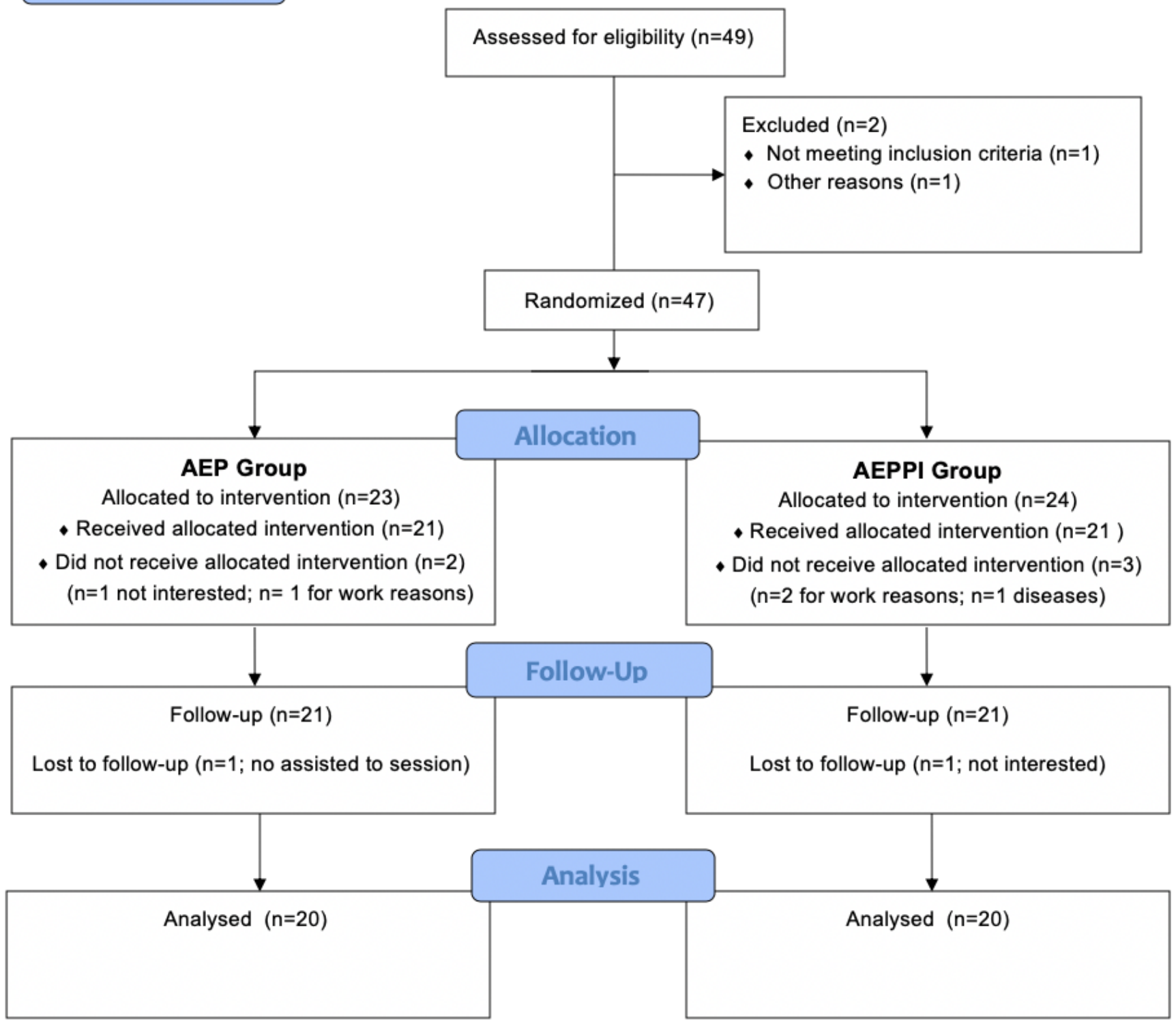

\section{Figure 1}

Flow diagram of participants. AEP, Abdominopelvic exercise program; AEPPI, Abdominopelvic exercise program adding postural instructions. 